\title{
MICRODILUTION PROCEDURE FOR ANTIFUNGAL SUSCEPTIBILITY TESTING OF Paracoccidioides brasiliensis TO AMPHOTERICIN B AND ITRACONAZOLE
}

\section{Takahagi-Nakaira E (1), Sugizaki MF (1), Peraçoli MTS (1)}

(1) Department of Microbiology and Immunology, Botucatu Biosciences Institute, São Paulo State University, UNESP, Botucatu, São Paulo State, Brazil.

\begin{abstract}
In vitro tests employing microdilution to evaluate fungal susceptibility to antifungal drugs are already standardized for fermentative yeasts. However, studies on the susceptibility of dimorphic fungi such as Paracoccidioides brasiliensis employing this method are scarce. The present work introduced some modifications into antifungal susceptibility testing from the European Committee on Antimicrobial Susceptibility Testing (EUCAST), concerning broth medium and reading time, to determine minimal inhibitory concentration (MIC) of amphotericin B and itraconazole against Paracoccidioides brasiliensis. Yeast-like cells of $P$. brasiliensis (Pb18 strain) were tested for susceptibility to amphotericin B and itraconazole in RPMI 1640 medium, supplemented with $2 \%$ glucose and nitrogen source and incubated at $35^{\circ} \mathrm{C}$. The MIC of amphotericin $\mathrm{B}$ and itraconazole against $\mathrm{Pb} 18$ were respectively 0.25 $\mu \mathrm{g} / \mathrm{mL}$ and $0.002 \mu \mathrm{g} / \mathrm{mL}$. The results of minimal fungicidal concentration (MFC) showed that amphotericin $B$ at $0.25 \mu \mathrm{g} / \mathrm{mL}$ or higher concentrations displayed fungicidal activity against $\mathrm{Pb} 18$ while itraconazole at least $0.002 \mu \mathrm{g} / \mathrm{mL}$ has a fungistatic effect on $P$. brasiliensis. In conclusion, our results showed that the method employed in the present study is reproducible and reliable for testing the susceptibility of $P$. brasiliensis to antifungal drugs.
\end{abstract}

KEY WORDS: amphotericin B, itraconazole, $P$. brasiliensis, antifungal susceptibility.

CONFLICTS OF INTEREST: There is no conflict.

FINANCIAL SOURCE: FAPESP.

\section{CORRESPONDENCE TO:}

MARIA TEREZINHA S. PERAÇOLI, Departamento de Microbiologia e Imunologia, Instituto de Biociências, UNESP, Botucatu, SP, 18618-970, Brasil. Fax: +55 $143815-$ 3744. Email: peracoli@ibb.unesp.br. 
Takahagi-Nakaira E et al. Microdilution procedure for antifungal susceptibility testing of Paracoccidioides brasiliensis to amphotericin B and itraconazole. J Venom Anim Toxins incl Trop Dis. 2009;15(4):719

\section{INTRODUCTION}

Paracoccidioidomycosis (PCM), a deep mycosis endemic to Latin America, is caused by Paracoccidioides brasiliensis, a fungus that presents thermal dimorphism and develops as yeast at body temperature (1). The clinical manifestations of the infection are equivalent to a chronic granulomatous disease with involvement of the lungs, reticuloendothelial system, mucocutaneous areas and other organs (2). The fungus enters the human body through inhalation of propagules that can only establish infection once they undergo phase transition to yeast in the pulmonary alveolar epithelium (3). Patients with acute or chronic PCM present symptoms such as malaise, fever, anorexia or weight loss tending to cachexia. In the severe forms of PCM, immune system alterations have been described including hypergammaglobulinemia, high level of specific antibodies, eosinophilia, and depressed general and antigen-specific cellular immunity (4-6).

The treatment of PCM is limited to the utilization of a few drugs including sulfonamides, amphotericin B and imidazole derivatives (ketoconazole, itraconazole and fluconazole). Since it was introduced for therapy, amphotericin B intravenous injection comprises an effective treatment that has been used for patients with severely disseminated mycosis $(7,8)$. This drug interacts with the plasma membrane of sensitive fungi, causing impairment of the barrier function and oxidative damage to the cell membrane. Its selectivity results from a higher affinity for the ergosterol component from fungal membranes than for cholesterol from mammalian cells (9, 10). The introduction of agents such as fluconazole and itraconazole (azole antifungal agents) has provided clinicians with less toxic oral alternatives to amphotericin B, marking a significant improvement in antifungal therapy (11).

Although methodologies for in vitro susceptibility testing have been available since the early years of antifungal drug development, few studies on antifungal susceptibilities of $P$. brasiliensis strains have been performed (12-15). In vitro tests of $P$. brasiliensis susceptibility to antimycotic drugs are scarce and the results have not always been consistent due to the diversity of the techniques employed $(13,14,16$ 18). However, significant progress has been achieved in relation to in vitro susceptibility testing, which aids in the initiation and monitoring of antifungal therapy $(19,20)$. The only standardization to determine the MIC is the reference method of the Clinical and Laboratorial Standards Institute (CLSI), adopted for yeasts, which has not been standardized for $P$. brasiliensis (21). 
The EUCAST techniques are characterized by their high reliability and reproducibility, are essential features for identifying organisms unlikely to respond to particular antifungal treatments (22). This standard is based on the CLSI reference procedure described in the document M27A2 (21).

In this investigation, we proposed a technique to determine the MIC of amphotericin $B$ and itraconazole against $P$. brasiliensis using the standard method of EUCAST, which employs RPMI 1640 medium supplemented with 2\% glucose for MIC determination by broth dilution of fermentative species of yeasts (22). Besides, we introduced a modification in this culture medium with the objective of providing a better growth of the fungus in microdilution plate wells and serial dilutions of antifungal agents. This alteration can contribute to the standardization of an adequate methodology for MIC determination of dimorphic fungi.

\section{MATERIALS AND METHODS}

\section{Fungus Strain}

The $P$. brasiliensis strain ( $\mathrm{Pb} 18$ ) was maintained in yeast cells at $35^{\circ} \mathrm{C}$ on RPMI 1640 without sodium bicarbonate (Gibco Laboratories, USA) and with L-glutamine (SigmaAldrich Inc., USA) buffered to $\mathrm{pH} 7.0$ with $0.165 \mathrm{M}$ morpholinepropanesulfonic acid (Sigma) and supplemented with $2 \%$ glucose, $2 \%$ ammonia sulfate, $0.12 \%$ asparagine (RPMI-Pb) and 2\% agar medium for the three days of culture. Yeast cells were washed and suspended in RPMI-Pb. In order to obtain individual cells, the fungal suspension was homogenized with glass beads in a vortex homogenizer (three cycles of ten seconds) and yeast cell viability was determined by phase-contrast microscopy $(23,24)$. Fungal suspensions containing more than $95 \%$ viable cells were used for the experiments.

\section{P. brasiliensis Growth}

After homogenization, yeast cell suspensions obtained as previously described, were dispensed into 96-well flat-bottomed sterile plastic microtiter plates (Corning Costar Europe, The Netherlands) at concentrations adjusted to $1 \times 10^{4}$ and $1 \times 10^{5}$ cells/well. The fungal growth was spectrophotometrically determined (Multiskan®, Eflab, Finland) by optical density reading of each well at $492 \mathrm{~nm}$ wavelength in different periods ranging from 0 to 168 hours at 12-hour intervals. 
Takahagi-Nakaira E et al. Microdilution procedure for antifungal susceptibility testing of Paracoccidioides brasiliensis to amphotericin B and itraconazole. J Venom Anim Toxins incl Trop Dis. 2009;15(4):721

\section{Antifungal Agents}

The azole derivative employed in the current study was itraconazole (Janssen, Belgium). The amphotericin B was purchased from Sigma. These drugs were obtained from their manufacturers as standard powders, each from a single lot. Stock solutions were prepared at concentrations 100 times the respective highest concentration to be tested, and were frozen in aliquots at $-70^{\circ} \mathrm{C}$ until use.

\section{Determination of Minimal Inhibitory Concentration (MIC)}

MIC determination followed EUCAST method by broth dilution of yeast fermentative species with minor modifications. Stock solutions were prepared in dimethyl sulfoxide while the culture medium was RPMI-Pb (22), prepared as a double-strength solution and sterilized by filtration. Sterile plastic microtiter plates containing flat-bottomed wells were utilized (Corning Costar). Each well contained $100 \mu \mathrm{L}$ of twofold serial dilutions of the antifungal drugs. Two drug-free medium wells were employed to provide sterility and growth controls. The final inoculum suspension contained $1 \times 10^{5}$ cells $/ \mathrm{mL}$, and a $100-\mu \mathrm{L}$ aliquot was added to each well of the microdilution plate. Final concentrations of amphotericin $B$ and itraconazole were serially diluted from 0.03 to $16 \mu \mathrm{g} / \mathrm{mL}$ and from 0.0001 to $16 \mu \mathrm{g} / \mathrm{mL}$, respectively. The microdilution plates were incubated at $35^{\circ} \mathrm{C}$ for 72 hours.

\section{Endpoint Determination}

The optical density of each microplate well was measured after 72 hours of incubation with a microplate spectrophotometer set at $492 \mathrm{~nm}$ wavelength (Multiskan $®$, Eflab, Finland). For itraconazole, the MIC endpoint was defined as the lowest drug concentration resulting in at least $50 \%$ growth reduction compared with controls, while that of amphotericin B was the lowest concentration that resulted in at least $90 \%$ growth reduction compared with the control.

\section{Determination of Minimal Fungicidal Concentration (MFC)}

After MIC determination, the microplates were shaken automatically for ten seconds. Then, $100 \mu \mathrm{L}$ was removed from each well showing growth inhibition and placed on supplemented brain-heart infusion (BHI) agar medium (Difco Laboratories, USA) plates containing $0.5 \%$ gentamicin, $4 \%$ horse normal serum, and $5 \% P$. brasiliensis 
strain 192 culture filtrate $(\mathrm{v} / \mathrm{v})$, the latter being the source of growth-promoting factor (25). For MFC determination, amphotericin B and itraconazole fungal suspensions were treated with antifungal concentrations ranging from $0.25 \times \mathrm{MIC}$ to $4 \times \mathrm{MIC}$ (0.0625 to $1 \mu \mathrm{g} / \mathrm{mL})$ and (0.0005 to 0.008$)$ respectively. A growth control culture without antifungals was submitted to the same procedures. Inoculated plates, of each culture in triplicate, were incubated at $35^{\circ} \mathrm{C}$ in sealed plastic bags to prevent drying. After 14 days, the number of colony forming units (CFU) per plate was counted using a colony counter (Quimis, Brasil). The MFC was defined as the lowest drug concentration from which $\leq 1$ colony was visible on the agar plate (26).

\section{Time-kill Curve Procedures}

Time-kill methods have provided information regarding rate and antifungal activity. The time-kill curve expresses the death rate of microorganisms produced at a fixed concentration of the antifungal drug. This rate was determined by the number of viable cells in periodic incubation intervals. The time-kill curve was obtained according to Klepser et al. (27) for Candida spp. to determine the required time of contact between fungi and antifungal drug necessary to kill $100 \%$ of $P$. brasiliensis yeast forms. For the MFC assay, concentrations of amphotericin B were added to the inoculum of $10^{4}$ yeast cells of $P$. brasiliensis. This suspension was incubated at $35^{\circ} \mathrm{C}$ and at $0,12,24,36,48,60,72,96$ and 120 hours of incubation; next, a 100- $\mu \mathrm{L}$ aliquot was plated on supplemented BHI-agar, as previously described. Culture controls without antifungal drug were submitted to the same procedures at the same intervals. All kill curves were performed in triplicate. For kill-curve studies, fungicidal activity was defined as CFU reduction of $\geq 3 \log 10$ (99.9\%) from the starting inoculum (28).

\section{Statistical Analysis}

Data were statistically analyzed by ANOVA using the GraphPad software package (GraphPad Instat ${ }$, version 3.05, USA) and compared by the Tukey-Kramer test, at the significance level $p<0.05$. 


\section{RESULTS}

The results of spectrophotometric evaluation of fungus growth showed that $1 \times 10^{4}$ yeast cells/well and 72 hours of incubation were, respectively, the best fungus concentration and incubation time to test for antifungal susceptibility. The fungus growth rate was confirmed by CFU recovery at different moments of culture ranging from 0 to 168 hours of incubation at $35^{\circ} \mathrm{C}$. At 72 hours, the highest growth rate was 3.9 times higher than those obtained at the initial culture time (time zero). This elevated growth was maintained up to 120 hours, after which, the fungus recovery diminished significantly (Table 1). Thus, 72-hour incubation was chosen since it revealed high viability after samples were plated on BHI agar medium. The MIC values for amphotericin $\mathrm{B}$ and itraconazole against $\mathrm{Pb} 18$ strain obtained by this procedure were respectively $0.25 \mu \mathrm{g} / \mathrm{mL}$ and $0.002 \mu \mathrm{g} / \mathrm{mL}$.

Table 1. Viable Paracoccidioides brasiliensis yeast cells recovery at different moments of culture

\begin{tabular}{ccc}
\hline Time of culture (h) & CFU $\times 10^{3}$ & Growth rate \\
\hline 0 & $4.60 \pm 1.40$ & 1.5 \\
12 & $6.68 \pm 0.67$ & 2.4 \\
24 & $10.9 \pm 0.64^{*}$ & 3.4 \\
36 & $15.6 \pm 1.63^{*}$ & 3.3 \\
48 & $15.18 \pm 0.41^{*}$ & 3.5 \\
60 & $16.13 \pm 0.6^{*}$ & 3.9 \\
72 & $17.94 \pm 1.10^{*+}$ & 3.7 \\
96 & $17.02 \pm 0.46^{*+}$ & 3.8 \\
120 & $17.6 \pm 0.41^{*+}$ & 3.1 \\
144 & $14.43 \pm 0.86^{*}$ & 3.4 \\
168 & $15.83 \pm 0.95^{*}$ &
\end{tabular}

Results are expressed as mean \pm SD of CFU from ten independent experiments ${ }^{*}(p<0.01)$ versus initial time, 12 hours; $+(p<0.01)$ versus $24,48,144,168$ hours (ANOVA) 
The antifungal effect of amphotericin B and itraconazole was determinated by MFC employing $P$. brasiliensis at MIC multiples ranging from $0.25 \times \mathrm{MIC}$ to $4 \times \mathrm{MIC}$. The fungal growth obtained was expressed in Figure 1. The results of MFC showed that amphotericin $B$ at $0.25 \mu \mathrm{g} / \mathrm{mL}$ or higher concentrations displayed significant fungicidal activity against $\mathrm{Pb} 18$ in a 72-hour culture (Figure $1 \mathrm{~A}$ ). This amphotericin $\mathrm{B}$ dose is coincident with the MIC concentration and inhibited $90 \%$ of $P$. brasiliensis growth. Thus, the amphotericin B concentration was established as MFC. Evaluation of MFC for itraconazole showed that the MIC value corresponding to $0.002 \mu \mathrm{g} / \mathrm{mL}$ led to $50 \%$ fungus growth inhibition that was maintained at higher antifungal concentrations (Figure 1B). These results indicate that itraconazole presents fungistatic effects on $P$. brasiliensis. Since the MFC for itraconazole was not obtained, time-kill studies for itraconazole were not performed.
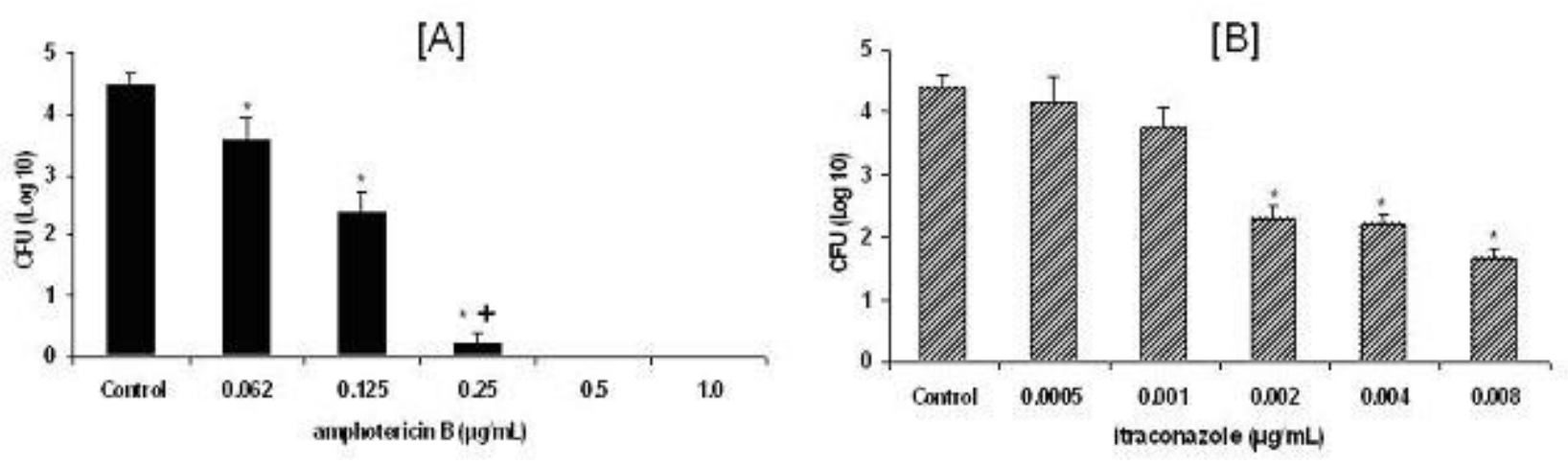

Figure 1. Viable yeast cells obtained from cultures with different $(A)$ amphotericin $B$ or (B) itraconazole concentrations. The results are expressed as mean log 10 $\mathrm{CFU} / \mathrm{mL}$ from ten independent experiments.

$[A]{ }^{*}(p<0.01)$ versus control; $+(p<0.01)$ versus $0.062,0.125$

$[B] *(p<0.01)$ versus control, $0.0005,0.001$

\section{Time-kill Curves}

Colony counts of $P$. brasiliensis as a function of time for amphotericin $B$ are presented in Figure 2. A pronounced concentration-dependent fungicidal activity was observed. The $0.5 \mu \mathrm{g} / \mathrm{mL}$ concentration had already killed $100 \%$ of the fungus at 36 hours, while at $0.25 \mu \mathrm{g} / \mathrm{mL}$ of amphotericin $B$, this effect was only observed at 120 hours. Thus, at $0.5 \mu \mathrm{g} / \mathrm{mL}$, amphotericin B exhibited fungicidal activity with a higher 
reduction in CFU/mL compared with the starting inoculum and control cultures without antifungals. P. brasiliensis yeast cells not treated with amphotericin B (control) showed constant growth from 0 to 120 hours of culture. The fungal growth in cultures with both amphotericin B concentrations was statistically different from control cultures in all periods of the antifungal treatment starting at 12 hours.
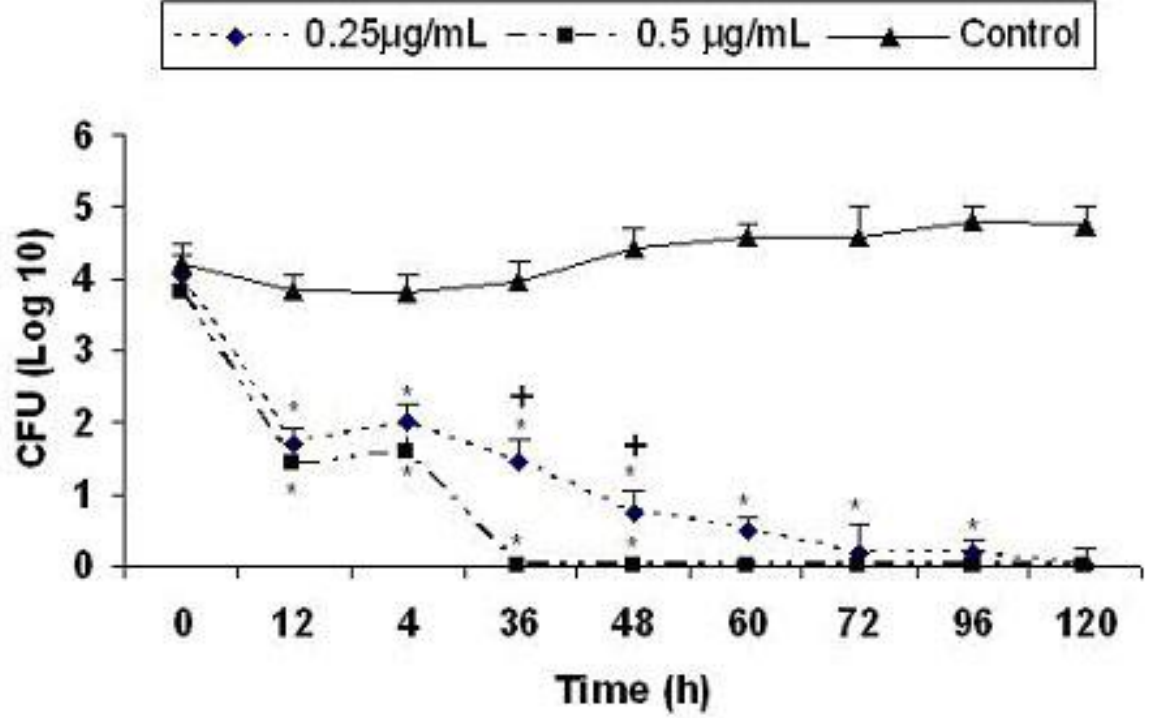

Figure 2. Time-kill plots showing the $P$. brasiliensis growth in cultures without or with $0.25 \mu \mathrm{g} / \mathrm{mL}$ and $0.5 \mu \mathrm{g} / \mathrm{mL}$ of amphotericin B. Results are representative of five independent experiments.

${ }^{*}(p<0.05)$ versus control; $+(p<0.05)$ versus $0.5 \mu \mathrm{g} / \mathrm{mL}$ (ANOVA)

\section{DISCUSSION}

In the present study, we proposed modifications on a broth microdilution method for testing susceptibility of $P$. brasiliensis to amphotericin B and itraconazole. So far, the MIC of amphotericin B and azoles for $P$. brasiliensis isolates were determined using broth macrodilution procedures (29-31).

Broth microdilution antifungal tests are similar to broth macrodilution tests; however, they require smaller amounts of medium and reagents, including the antifungal drugs. Even though antifungal broth microdilution tests are not as widely used as broth macrodilution assays, some laboratories employ them more frequently with yeasts because their cost is lower and they are less cumbersome (32-34). 
Takahagi-Nakaira E et al. Microdilution procedure for antifungal susceptibility testing of Paracoccidioides brasiliensis to amphotericin B and itraconazole. J Venom Anim Toxins incl Trop Dis. 2009;15(4):726

The EUCAST evaluation for yeasts is based on the CLSI reference procedure described in the document M27-A2 (21), but includes some modifications to allow for automation of the method and permit the incubation period to be shortened from 48 to 24 hours, thus decreasing the subjectivity and increasing the sensitivity of the assay (35). A multicenter evaluation has demonstrated that the EUCAST procedure for antifungal susceptibility testing is a reproducible method, with $94 \%$ agreement among laboratories (22). Nonetheless, this methodology has not yet been established to test antifungal drugs for dimorphic fungi. As RPMI 1640 has not been considered a medium suitable for studies of $P$. brasiliensis growth, and McVeighMorton (MVM) medium provided efficient fungus growth, we compared the two medium chemical compositions and supplemented RPMI 1640 with 2\% glucose and nitrogen sources such as asparagine and ammonium sulfate (30). Thus, we proposed these modifications in RPMI 1640 medium and a inoculum size of $1 \times 10^{4}$ cells, and observed a prolific growth of $P$. brasiliensis to MIC determination.

The growth medium composition can significantly influence growth characteristics of fungi. The impact of medium selection is evident not only on the rate and degree of growth, but also on the apparent in vitro susceptibility of isolates to various antifungal agents (17). Furthermore, previous studies indicated that glucose supplementation, large inoculum size, and spectrophotometric reading may serve as a less subjective method than visual assessment to evaluate fungal growth in the presence of antifungal agents (36-38). RPMI supplemented with glucose has been indicated as aiding in the growth of yeasts and may simplify endpoint determination due to higher turbidity of the growth control $(37,39,40)$.

The results obtained with amphotericin $B$ and itraconazole are similar to those reported by other authors that employed different techniques and other culture media to evaluate the $P$. brasiliensis sensibility to antifungals in vitro (29-31). Nakai et al. (15) evaluated fungicidal activity of micafungin against dimorphic fungi, including $P$. brasiliensis, by comparing it with other antifungals such as amphotericin $B$, itraconazole and fluconazole. The results demonstrated that the MIC of amphotericin B ranged from 0.0078 to $0.25 \mu \mathrm{g} / \mathrm{mL}$, while the MIC of itraconazole was lower than $0.0039 \mu \mathrm{g} / \mathrm{mL}$. Even though these authors utilized the visual method to evaluate MIC, the results were similar to those obtained in the present study employing spectrophotometric method. Although these works demonstrated agreement between 
results obtained by visual and espectrophotometric methods, the latter offers an advantage by providing more objective and automated MIC determination.

MFC, using amphotericin $B$, has been employed to evaluate the connection between the drug concentration and its antifungal activity against the microorganism. Different authors have provided various definitions of MFC, including the lowest drug concentration that permitted the growth of: 3 colonies, $\leq 5$ colonies, $\leq 10$ colonies (macrodilution) or $\leq 1$ colony (microdilution) visible on agar plates $(26,41,42)$. According to the EUCAST, MFC is defined as a one-thousand-fold reduction in CFU compared to the control value (22). In the present study, we defined MFC as the lowest amphotericin $B$ concentration at which $\leq 10$ colonies were visible on the plate. The time-kill curve method confirmed that amphotericin B at $0.25 \mu \mathrm{g} / \mathrm{mL}$ concentration displayed fungicidal activity against $P$. brasiliensis in a 72-hour culture. Additionally, the time-kill curve revealed a dynamic relationship between amphotericin $B$ and its effects on $P$. brasiliensis.

Therefore, the present findings suggest that it is possible to use more efficient and economical broth microdilution to test antifungal drugs against $P$. brasiliensis. The spectrophotometric method offers an advantage over the visual method by providing a more objective and automated MIC determination. In conclusion, the methodology employed in the current study is easy to perform, economical, reproducible and reliable for testing the susceptibility of $P$. brasiliensis to antifungal drugs.

\section{ACKNOWLEDGEMENTS}

This work was supported by The State of São Paulo Research Foundation, FAPESP, Brazil (grants 02/12462-4 and 03/12743-0).

\section{REFERENCES}

1. Restrepo A, Tobón A. Paracoccidioides brasiliensis. In: Mandell GL, Bennettt JE Dollin R, editors. Principles and practice of infectious diseases. $6^{\text {th }}$ ed. Philadelphia: Elsevier; 2005. p. 3062-68.

2. Franco MF, Mendes RP, Moscardi-Bacchi M, Montenegro MR. Paracoccidioidomycosis. Bailliere's Clin Trop Commun Dis. 1989;4:185-220.

3. McEwen JG, Bedoya V, Patiño MM, Salazar ME, Restrepo AM. Experimental murine paracoccidiodomycosis induced by the inhalation of conidia. $\mathrm{J}$ Med Vet Mycol. 1987;25(3):165-75. 
4. Bernard G, Mendes-Giannini MJ, Juvenale M, Miranda ET, Duarte AJ. Immunosuppression in paracoccidioidomycosis: T cell hyporesponsiveness to two Paracoccidioides brasiliensis glycoproteins that elicit strong humoral immune response. J Infect Dis. 1997;175(5):1263-7.

5. Mendes RP. The gamut of clinical manifestations. In: Franco M, Lacaz CS, Restrepo-Moreno A, Del Negro G, editors. Paracoccidioidomycosis. Boca Raton: CRC Press; 1994. p. 33-58.

6. Musatti CC, Peracoli MTS, Soares AMVC, Rezkallah-Iwasso MT. Cell mediated immunity in patients with paracoccidioidomycosis. In: Franco M, Lacaz CS, RestrepoMoreno A, Del Negro G, editors. Paracoccidioidomycosis. Boca Raton: CRC Press; 1994. p. $175-86$.

7. Dewsnup DH, Stevens DA. Efficacy of oral amphotericin B in AIDS patients with thrush clinically resistant to fluconazole. J Med Vet Mycol. 1994;32(5):389-93.

8. Gallis HA, Drew RH, Pickard WW. Amphotericin B: 30 years of clinical experience. Rev Infect Dis. 1990;12(2):308-29.

9. Georgopapadakou NH, Tkacz J. The fungal cell wall as a drug target. Trends Microbiol. 1995;3(3):98-104.

10. Shadomy S, Espinel-Ingroff A. Susceptibility testing with antifungal drugs. In: Lennette EH, Balows A, Hausler WJ, Truant JP, editors. Manual of clinical microbiology. $3^{\text {rd }}$ ed. Washington; American Society for Microbiology; 1980. p. 64753.

11. Ernst ME, Klepser ME, Wolfe EJ, Pfaller MA. Antifungal dynamics of LY 303366 , an investigational echinocandin B analog, against Candida ssp. Diagn Microbiol Infect Dis. 1996;26(3-4):125-31.

12. Shadomy S. In vitro studies with 5-fluorocytosine. Appl Environ Microbiol. 1969;17(6):871-7.

13. Hamdan JS, de Resende MA. Lipid composition and effect of amphotericin $B$ on yeast cells of Paracoccidioides brasiliensis. Mycopathologia. 1988;102(2):97-105.

14. Jesuino RS, Soares RB, Salem-Izacc SM, Pereira M, Felipe MS, Soares CM. In vitro amphotericin B effects on growth, viability and dimorphism of Paracoccidioides brasiliensis: reversal of the treatment. Microbiol Immunol. 1997;41(2):93-9.

15. Nakai T, Uno J, Ikeda F, Tawara S, Nishimura K, Miyaji M. In vitro antifungal activity of Micafungin (FK463) against dimorphic fungi: comparison of yeast-like and mycelial forms. Antimicrob Agents Chemother. 2003;47(4):1376-81. 
Takahagi-Nakaira E et al. Microdilution procedure for antifungal susceptibility testing of Paracoccidioides brasiliensis to amphotericin B and itraconazole. J Venom Anim Toxins incl Trop Dis. 2009;15(4):729

16. Heins-Vaccari E, Takahashi de Melo N, da Silva Lacaz C, Dias Pereira A, Del Negro G. Ação in vitro do itraconazol (R-51211) sobre Paracoccidioides brasiliensis, Histoplasma capsulatum var. capsulatum e Histoplasma capsulatum var. duboisii. An Bras Dermat. 1988;63(3):299-302.

17. Pfaller MA, Rinaldi MG, Galgiani JN, Bartlett MS, Body BA, Espinel-Ingroff A, et al. Collaborative investigation of variables in susceptibility testing of yeasts. Antimicrob Agents Chemother. 1990;34(9):1648-54.

18. San-Blas G, Calcagno AM, San-Blas F. A preliminary study of in vitro antibiotic activity of saperconazole and other azoles on Paracoccidioides brasiliensis. J Med Vet Mycol. 1993;31(2):169-74.

19. Espinel-Ingroff A, Shadomy S. In vitro and in vivo evaluation of antifungal agents. Eur J Clin Microbiol Infect Dis. 1989;8(4):352-61.

20. Shadomy S, Espinel-Ingroff A, Cartwright R. Laboratory studies with antifungal agents: susceptibility tests and bioassays. In: Lennette EH, Balows A, Hausler Jr WJ, Shadomy HJ, editors. Manual of clinical microbiology. $4^{\text {th }}$ ed. Washington: American Society for Microbiology; 1985. p. 991-9.

21. National Committee for Clinical Laboratory Standards (NCCLS). Reference method for broth dilution antifungal susceptibility testing of yeasts. Approved standard - second edition M27-A2. Wayne: National Committee for Clinical Laboratory Standards; 2002.

22. Subcommittee on Antifungal Susceptibility Testing (AFST) of the ESCMID European Committee for Antimicrobial Susceptibility Testing (EUCAST). EUCAST definitive document EDef 7.1: method for the determination of broth dilution MICs of antifungal agents for fermentative yeasts. Clin Microbiol Infect. 2008;14(4):398-405.

23. Peraçoli MT, Sugizaki MF, Mendes RP, Naiff R, Montenegro MR. Paracoccidioides brasiliensis isolated from armadillos is virulent to Syrian hamsters. Mycopathologia. 1999;148(3):123-30.

24. Soares AM, Calvi SA, Peraçoli MT, Fernadez AC, Dias LA, Dos Anjos AR. Modulatory effect of prostaglandins on human monocyte activation for killing of highand low-virulence strains of Paracoccidioides brasiliensis. Immunology. 2001;102(4):480-5.

25. Singer-Vermes LM, Ciavaglia MC, Kashino SS, Burger E, Calich VL. The source of the growth-promoting factor(s) affects the plating efficiency of Paracoccidioides brasiliensis. J Med Vet Mycol. 1992;30(3):261-4. 
26. Pujol I, Aguilar C, Fernandez-Ballart J, Guarro J. Comparison of the minimum fungicidal concentration of amphotericin B determined in filamentous fungi by macrodilution and microdilution methods. Med Mycol. 2000;38(1):23-6.

27. Klepser ME, Ernst EJ, Lewis RE, Ernst ME, Pfaller MA. Influence of test conditions on antifungal time-kill curve results: proposal for standardized methods. Antimicrob Agents Chemother. 1998;42(5):1207-12.

28. Pearson RD, Steigbigel RT, Davis HT, Chapman SW. Method of reliable determination of minimal lethal antibiotic concentrations. Antimicrob Agents Chemother. 1980;18(5):699-708.

29. Hahn RC, Hamdan JS. Effects of amphotericin B and three azole derivatives on the lipids of yeast cells of Paracoccidioides brasiliensis. Antimicrob Agents Chemother. 2000;44(7):1997-2000.

30. Hahn RC, Fontes CJ, Batista RD, Hamdan JS. In vitro comparison of activities of terbinafine and itraconazole against Paracoccidioides brasiliensis. J Clin Microbiol. 2002;40(8):2828-31.

31. Restrepo A, Tabares CB. In vitro susceptibility of Paracoccidioides brasiliensis yeasts form to antifungal agents. Rev Inst Med Trop São Paulo. 1984;26(6):322-8.

32. Guinet R, Nerson D, de Closets F, Dupouy-Camet J, Kures L, Marjollet M, Poirot JL, Ros A, Texier-Maugein J, Volle PJ. Collaborative evaluation in seven laboratories of a standardized micromethod for yeast susceptibility testing. J Clin Microbiol. 1988;26(11):2307-12.

33. Hector RF, Zimmer BL, Pappagianis D. Microtiter method for MIC testing with spherule-endospore-phase Coccidioides immitis. J Clin Microbiol. 1988;26(12):26678.

34. Radetsky M, Wheeler RC, Roe MH, Todd JK. Microtiter broth dilution method for yeast susceptibility testing with validation by clinical outcome. J Clin Microbiol. 1986;24(4):600-6.

35. Cuenca-Estrella M, Gomez-Lopez A, Mellado E, Rodriguez-Tudela JL. Correlation between the procedure for antifungal susceptibility testing for Candida spp. of the European Committee on Antibiotic Susceptibility Testing (EUCAST) and four commercial techniques. Clin Microbiol Infect. 2005;11(6):486-92. 
36. Lozano-Chiu M, Arikan S, Paetznick VL, Anaissie EJ, Rex JH. Optimizing voriconazole susceptibility testing of Candida: effects of incubation time, endpoint rule, species of Candida, and level of fluconazole susceptibility. J Clin Microbiol. 1999;37(9):2755-9.

37. Odds FC, Vranckx L, Woestenborghs F. Antifungal susceptibility testing of yeasts: evaluation of technical variables for test automation. Antimicrob Agents Chemother. 1995;39(9):2051-60.

38. Rodriguez-Tudela JL, Martinez-Suárez JV. Defining conditions for microbroth antifungal susceptibility tests: influence of RPMI and RPMI-2\% glucose on the selection of endpoint criteria. J Antimicrob Chemother. 1995;35(6):739-49.

39. Espinel-Ingroff A, Rodríguez-Tudela JL, Martínez-Suárez JV. Comparison of two alternative microdilution procedures with the National Committee for Clinical Laboratory Standards reference macrodilution method M27-P for in vitro testing of fluconazole-resistant and -susceptible isolates of Candida albicans. J Clin Microbiol. 1995;33(12):3154-8.

40. Cuenca-Estrella M, Díaz-Guerra TM, Mellado E, Rodríguez-Tudela JL. Influence of glucose supplementation and inoculum size on growth kinetics and antifungal susceptibility testing of Candida spp. J Clin Microbiol. 2001;39(2):525-32.

41. Li RK, Ciblak MA, Nordoff N, Pasarell L, Warnock DW, McGinnis MR. In vitro activities of voriconazole, itraconazole, and amphotericin $B$ against Blastomyces dermatitidis, Coccidioides immitis, and Histoplasma capsulatum. Antimicrob Agents Chemother. 2000;44(6):1734-6.

42. Sutton DA, Sanche SE, Revankar SG, Fothergill AW, Rinaldi MG. In vitro amphotericin $\mathrm{B}$ resistance in clinical isolates of Aspergillus terreus, with a head-tohead comparison to voriconazole. J Clin Microbiol. 1999;37(7):2343-5. 